Article

\title{
Enhancement of Lignin Extraction of Poplar by Treatment of Deep Eutectic Solvent with Low Halogen Content
}

\author{
Jinke Liu ${ }^{1, \dagger}$, Letian $\mathrm{Qi}^{1,+}{ }^{1}$, Guihua Yang ${ }^{1, *} \mathbb{C}$, Yu Xue ${ }^{1}$, Ming He ${ }^{1 \mathbb{C}}$, Lucian A. Lucia ${ }^{1,2}$ \\ and Jiachuan Chen ${ }^{1}$ \\ 1 State Key Laboratory of Biobased Material and Green Papermaking, Qilu University of Technology, \\ Shandong Academy of Sciences, Jinan 250353, China; ljk810319@gmail.com (J.L.); lqi01@qlu.edu.cn (L.Q.); \\ xueyu@qlu.edu.cn (Y.X.); heming8916@qlu.edu.cn (M.H.); lucian.lucia@ncsu.edu (L.A.L.); \\ chenjc@qlu.edu.cn (J.C.) \\ 2 Department of Forest Biomaterials, North Carolina State University, Box 8005, Raleigh, NC 27695-8005, USA \\ * Correspondence: ygh@qlu.edu.cn; Tel.: +86-531-8963-1884 \\ + These authors have contributed equally to this work.
}

Received: 16 June 2020; Accepted: 16 July 2020; Published: 18 July 2020

\begin{abstract}
A novel choline-based deep eutectic solvent (DES) with low halogen content-namely choline lactate-lactic acid (CLL) — was synthesized by replacing the chloride anion with lactate anion in choline chloride-lactic acid (CCL). CLL and CCL treatments were conducted at $140{ }^{\circ} \mathrm{C}$ for $12 \mathrm{~h}$ with hydrogen bond acceptor/hydrogen bond donor $=1 / 10$, thereafter composition analysis and characterizations of the lignin extracted by DES treatment (DES lignin) and the solid residue were carried out. The proposed low halogen content DES presented an improved lignin extraction efficiency. The CLL treatment extracted $90.13 \%$ of initial lignin from poplar, while CCL extracted $86.02 \%$. In addition, the CLL treatment also provided DES lignin with an improved purity $(91.17 \%)$, lower molecular weight $(\mathrm{Mw} / \mathrm{Mn}=1805 / 971 \mathrm{~g} / \mathrm{mol}$ ) and more concentrated distribution (polydispersity index=1.86). The efficient lignin extraction was mainly ascribed to the cleavage of $\beta-\mathrm{O}-4$ bonds in lignin macromolecule, especially in the guaiacyl units, thereby breaking them into smaller molecules, facilitating the lignin extraction. The replacement of chloride anion allowed CLL acting as a more efficient DES to interact with lignin macromolecules, thus providing lignin with higher uniformity and suitable molecular weight. The low halogen content DES system proposed in present work could benefit the fractionation of biomass, improve the valorization of lignin compounds and facilitate industrial process in the downstream.
\end{abstract}

Keywords: lignin extraction; deep eutectic solvent; low halogen content; poplar; treatment

\section{Introduction}

Lignocellulosic biomass is a promising carbon-based natural alternative to fossil fuel resources due to its abundant availability, low cost and renewable feature [1]. The utilization and conversion of this sustainable biomass attracted the interest from both academic and industry [2]. Lignocelluloses are mainly composed by cellulose $(40 \%)$, hemicellulose $(25 \%)$ and lignin $(25 \%)$. Among them the cellulose and hemicellulose are sugar polymers, whereas lignin is highly crosslinked and branched amorphous polymer [3]. The cellulose and hemicellulose can be easily converted into sugars and biofuels through the hydrolysis and fermentation process, which have been widely reported [4,5]. During the conversion process state above, lignin is considered as an inhibitor during the conversion of carbohydrates for its massive recalcitrant structure [6]. While, from the other point of view, lignin is also the most abundant renewable aromatic polymer on earth [7] and presents in all higher plants 
as an integral part of the plant fiber cell wall $[7,8]$. Thereby, the investigation of lignin extraction benefits both the valorization of lignin and the processing of cellulose and hemicellulose products. However, due to the rigid structure of plant fibers and complex crosslinks between the components [9], the lignin cannot be separated directly and efficiently without significant modification of its native structure [10]. Pretreatments are commonly used, among which the extraction of lignin with ordinary solvents have been investigated extensively and reviewed in detail elsewhere [11-13].

The recent discovery and development of deep eutectic solvent (DES) as an effectively solvent for the lignin extraction provided a new insight through this field [14]. The related DES was first, induced by Abbott [15] as a sustainable, biodegradable and designable solvent with great potential for varies applications [16-18]. In addition to its tunable and environmentally friendly feature, their strong hydrogen bonding (HB) ability presents unique solubility to the biomass, which could facilitate the selective extraction of lignin from lignocellulosic biomass [7,19]. Effective DES structures were reported being composed by tetraalkylammonium-based hydrogen bond acceptors (HBA), mixed with various hydrogen bond donors (HBD), including alcohols, carboxylic acids and amino acids. It is believed that the interactions between HBA and HBD reduce the electrostatic force between the cation and anion. Thereby, the formation of HBs in DES mixtures could reduce the freezing point of the mixture and facilitate the selective extraction of lignin compounds. The literatures [20-22] clearly demonstrated that choline-based DES could efficiently extract lignin from lignocellulosic, while choline chloride-lactic acid is one of the most efficient DES to extract lignin with high yield and high purity. The depolymerization mechanism of lignin was reported that DES selectively cleaved the ether linkages in wood fibers and facilitated the lignin extraction from lignocellulosic biomass.

Choline chloride $([\mathrm{Ch}][\mathrm{Cl}])$ and its alternatives were also applied in the fractionating of lignocellulosic biomass. Their solvation chemistry of was mainly determined by their anion while the cation having a secondary effect [23]. Choline carboxylic acid [24,25] and choline amino acid [26-30] were synthesized and reported as effective solvents for lignocellulosic biomass pretreatment. In addition, these choline-based ionic liquids also presented low toxicity and good biodegradability [25,31]. These choline-based ionic liquids (ILs) could selectively dissolve and extract lignin. In addition, the removal of chloride anion in choline-based ILs could avoid the corrosion of process equipment [32]. Replacing the chloride anion in choline chloride-lactic acid DES with carboxylate could improve the selectivity during the lignin extraction and prevent the corrosion of process equipment.

In the present work, a novel choline-based DES-namely, choline lactate-lactic acid (CLL) - was synthesized by replacing the chloride anion in choline chloride-lactic acid (CCL). Both CLL and CCL were used to selectively separate lignin from poplar for evaluating the extraction efficiency of CLL and CCL. The purity and structural characterization of lignin were analyzed by gel permeation chromatography (GPC), Fourier-transform infrared (FT-IR) and heteronuclear single quantum coherence nuclear magnetic resonance (HSQC NMR).

\section{Materials and Methods}

\subsection{Materials}

Poplar chips were obtained from Sun Paper Co., Ltd. (Jining, China). Wood chips were selected with uniform size and grinded in a star mill. The mesh was sifted through 40-60 sieve to obtain the wood powder. Subsequently, the wood powder was extracted with benzene-alcohol solution for $8 \mathrm{~h}$. The dry wood powder after the extraction was used as the lignocellulose raw material for this experiment and its chemical composition was measured. The raw hardwood sample (poplar) contains $45.46 \%$ glucose, $14.08 \%$ xylose, $1.71 \%$ mannose, $0.41 \%$ galactose, $0.21 \%$ arabinose and $25.62 \%$ lignin.

\subsection{Chemicals}

Choline chloride ([Ch][Cl]), ethanol, benzene, pyridine acetic anhydride and diethyl ether were purchased from Sinopharm Chemical Reagent Co., Ltd. (Shanghai, China). lactic acid and potassium 
bromide (KBr) were from Tianjin Kemiou Chemical Reagent Co., Ltd. (Tianjin, China); tetrahydrofuran (THF) and potassium hydroxide (KOH) were from Tianjin HengXing Chemical Reagent Co., Ltd. (Tianjin, China); dimethyl sulfoxide $\left(\mathrm{DMSO}_{6} \mathrm{~d}_{6}\right.$ ) were from Shanghai Macklin Biochemical Technology Co., Ltd. (Shanghai, China).

\subsection{Preparation of DES}

The choline lactate ([Ch][Lac]) was synthesized according to the literature method [33]. [Ch][Cl] and sodium hydroxide were mixed in ethanol. The mixture was kept at in $60^{\circ} \mathrm{C}$ water bath for $4 \mathrm{~h}$. Subsequently, the supernatant of mixture was filtered and added with lactic acid. The solvent in the supernatant was then removed by rotary evaporation under reduced pressure to obtain [Ch][Lac].

Choline chloride-lactic acid (CCL) and choline lactate-lactic acid (CLL) used in this work were synthesized by mixing lactic acid with choline chloride and choline lactate, respectively. The DES was synthesized with seven different HBA to HBD ratios (molar ratio 1/2, 1/4, 1/6, 1/8, 1/10, 1/12, 1/14).The mixture was stirred at $65^{\circ} \mathrm{C}$ for $2 \mathrm{~h}$ to generate a well-mixed, homogeneous and transparent liquid. The obtained CCL and CLL were cooled down to room temperature in a desiccator for the further application.

The general physical parameters, including melting point $\left(\mathrm{T}_{\mathrm{m}}\right), \mathrm{pH}$, viscosity of CCL and CLL are listed in Table 1.

Table 1. General physical parameters of novel choline lactate-lactic acid (CLL) and choline chloride-lactic acid (CCL).

\begin{tabular}{lcccc}
\hline DES & $\mathbf{T}_{\mathbf{m}}\left({ }^{\circ} \mathbf{C}\right)$ & Viscosity (Pa.s) & Density $\left(\mathrm{g} / \mathrm{cm}^{3}\right)$ & $\mathbf{p H}$ \\
\hline CCL & $18.34 \pm 0.34$ & 0.87 & 1.2022 & 0.91 \\
CLL & $18.14 \pm 0.39$ & 1.38 & 1.2078 & 2.75 \\
\hline
\end{tabular}

\subsection{DES Treatment}

DES treatment for lignin extraction was processed according to the literature method [7], where $1.5 \mathrm{~g}$ over-dry biomass and $45 \mathrm{~g}$ DES were mixed in a $100 \mathrm{~mL}$ pressure vessel, which was incubated at $80-160{ }^{\circ} \mathrm{C}$ for $4-14 \mathrm{~h}$. The reaction was terminated by adding large amount of ethanol. The solid residue was separated by filtration, washed by ethanol and water, then dried in oven at $105^{\circ} \mathrm{C}$. Deionized water (DI water) was added into the filtrate to precipitate the extracted lignin by DES treatment (DES lignin). Lignin was collected by centrifugation and washed with additional water/ethanol mixture for purification. Composition analyses of solid residue and DES lignin were conducted following the standard NREL procedures [34]. The process of DES treatment for lignin extraction is shown in Figure 1.

The HBA/HBD ratio, process temperature and duration for lignin extraction conditions were optimized by experiments. The optimized DES treating conditions were obtained with HBA/HBD of $1 / 10$, operating at $140{ }^{\circ} \mathrm{C}$ for $12 \mathrm{~h}$. The optimal conditions were selected for further investigation in this work, in which every sample was tested at least three times and the average value was taken and reported.

The recovery of DES was carried out following literature method [35]. Briefly, the effluent generated by water and/or ethanol washing was collected and filtered to remove insoluble content. The filtrate was collected and dehydrated under reduced pressure. Repeat the filtration and dehydration process until the filtrate became a clear solution. Further dehydration was performed under reduced pressure with Schlenk line at $70{ }^{\circ} \mathrm{C}$ for $24 \mathrm{~h}$. The structure and purity of the recycled DES were checked by NMR. 


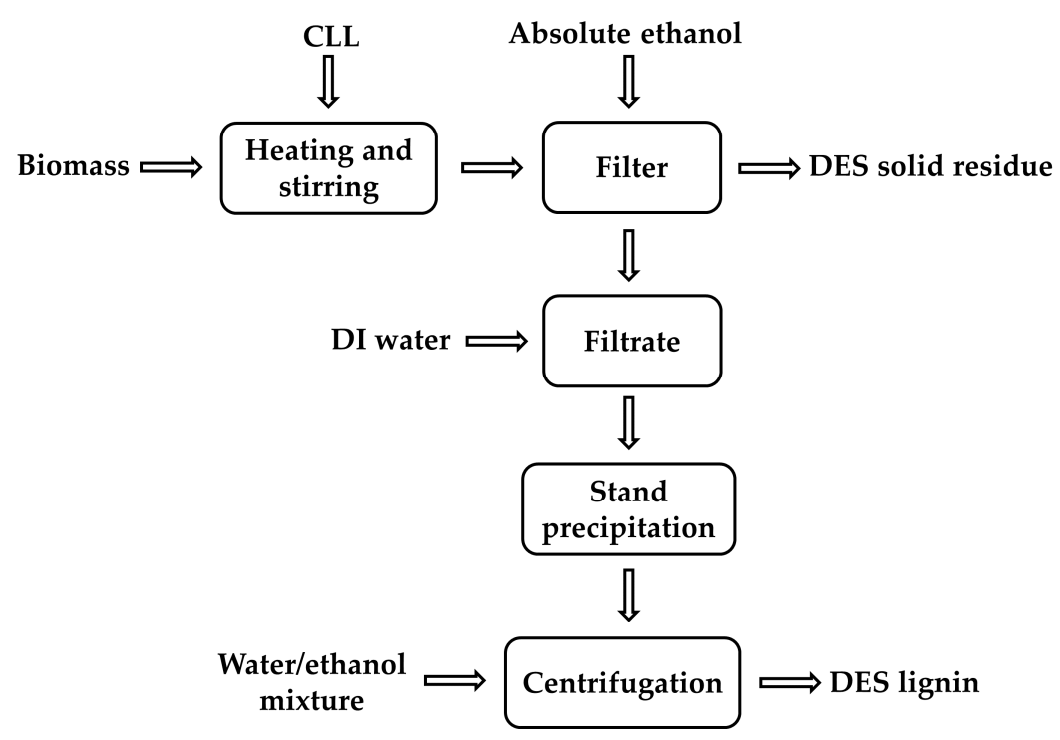

Figure 1. The process of lignin extraction from lignocellulosic biomass by deep eutectic solvent (DES) treatment.

\subsection{Preparation of Milled Wood Lignin}

Milled wood lignin (MWL) was isolated from the poplar wood species according to the procedure reported by Björkman [36]. Briefly, poplar powder was put into a ball mill for $72 \mathrm{~h}$ at room temperature. Subsequently, the powder was extracted with a dioxane-water mixture and centrifuged to obtain a supernatant. The supernatant was put onto rotary evaporator to remove solvents and then freeze-dried to obtain the MWL product.

\subsection{Analysis of Lignin Characteristics}

\subsubsection{FT-IR Analysis}

FT-IR (VERTEX70, Bruker, Karlsruhe, Germany) was used to analyze lignin characteristics. The dried samples were embedded in KBr pellets at concentrations of $1 \mathrm{mg} / 100 \mathrm{mg} \mathrm{KBr}$. All spectra were recorded in absorption band mode over the range of 4000 to $500 \mathrm{~cm}^{-1}$.

\subsubsection{Acetylation of Lignin}

For the acetylation of lignin samples, 50-mg lignin sample was dissolved in $2 \mathrm{~mL}$ pyridine in a $20-\mathrm{mL}$ glass vial, followed by the addition of acetic anhydride $(2 \mathrm{~mL})$. The mixture was sealed and allowed to react at room temperature for $24 \mathrm{~h}$. The acetylated lignin was precipitated in ice DI-water with constant stirring and collected by filtration. The collected acetylated lignin was washed with DI-water and vacuum-dried.

\subsubsection{GPC Analysis}

The molecular weight distribution of lignin samples was determined by GPC (WATERS Alliance HPLC e2695, Waters, MA, USA). Approximately $2 \mathrm{mg}$ of acetylated lignin sample was dissolved in tetrahydrofuran and then filtered through a $0.45-\mu \mathrm{m}$ filter. An Agilent 1200 series high performance liquid chromatography (HPLC) equipped with an ultraviolet detector (UV) at $254 \mathrm{~nm}$ was used to conduct GPC analysis of lignin molecular weight distribution. Polystyrene standards with molecular weights ranging from 139 to $16,000 \mathrm{~g} / \mathrm{mol}$ were used to calibrate the molecular weight based on retention time. 


\subsubsection{HSQC NMR Analysis}

NMR (AVANCE III 500 MHz, Bruker, Karlsruhe, Germany) were recorded according to literature method [7], by using a spectrometer equipped with a DCH cryoprobe. HSQC spectra was recorded at $25{ }^{\circ} \mathrm{C}$ using the Q-CAHSQC pulse program. Matrices of 2048 data points for the ${ }^{1} \mathrm{H}$-dimension $(13$ to $-1 \mathrm{ppm})$ and 1024 data for the ${ }^{13} \mathrm{C}$-dimension (160 to $0 \mathrm{ppm}$ ) were collected, with the relaxation delay set at $6 \mathrm{~s}$ [7]. The lignin samples were dissolved in $0.5 \mathrm{~mL}$ of dimethylsulfoxide-d6 $\left(\mathrm{DMSO}^{\mathrm{d}} \mathrm{d}_{6}\right)$ and chemical shifts were referenced to the solvent signal (2.50/40.21 ppm).

\section{Results and Discussion}

\subsection{Lignin Extracted by Choline-Based DES}

In order to evaluate the efficiency of choline base-DES on the lignin extraction from the poplar, CCL and CLL at HBA/HBD of 1/10 (molar ratio) were prepared. The treatment was carried out at temperature of $140{ }^{\circ} \mathrm{C}$ and solid ratio of 30:1 (mass ratio) for $12 \mathrm{~h}$. The sugars and lignin of samples with or without DES treatment were analyzed. The distribution of the main chemical compositions in DES solid residue and DES lignin are shown in Figure 2, in which the calculation was based on the dry initial sample. The whiskers regions in the figure indicate the compositions extracted into DES lignin, while black bars are corresponding to compositions remain in the solid residues. It could be seen that DES treatment efficiently fractionated the components of poplar samples by the lignin removal, while keeping the cellulose content in the solid residues. The raw sample contains $45.46 \%$ glucose, $14.08 \%$ xylose, $1.71 \%$ mannose and $25.62 \%$ lignin. While in the CCL treatment process, $22.04 \%$ of native lignin was extracted into CCL lignin phase and $43.37 \%$ of cellulose (glucose) was kept in the solid residues. It was intriguing to find CLL treatment presented higher fractionation efficiency, as a higher amount of lignin presented in CLL lignin (23.09\%), alongside with more cellulose component (44.73\%) in CLL solid residue. It should be noticed that a large amount of mannose $(1.14 \%)$ was also preserved in CLL solid residue, which was not found in CCL-treated samples. Therefore, the CLL seems to be a promising solvent to achieve the selective separation of lignin from lignocellulosic biomass.

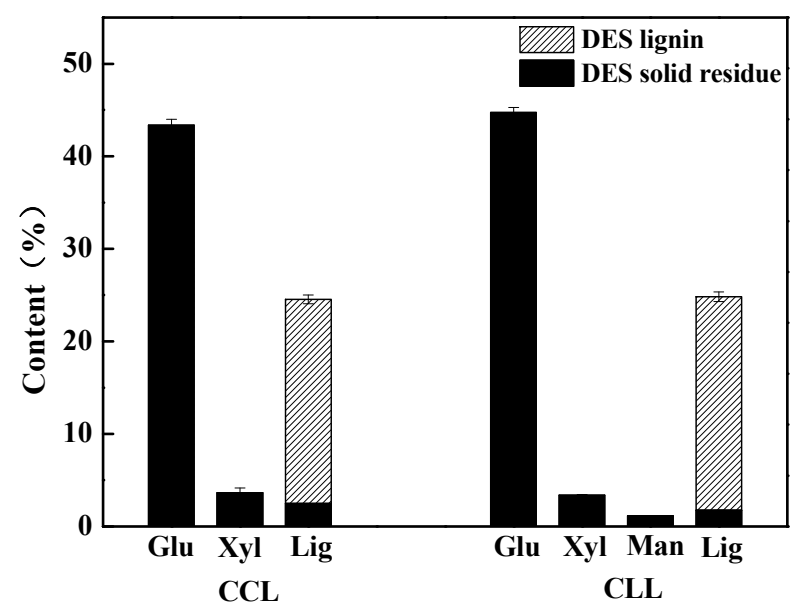

Figure 2. Composition analysis of samples in DES-treated solid residue and DES lignin at $140{ }^{\circ} \mathrm{C}$ for $12 \mathrm{~h}$. (Based on over-dry basis of the initial sample).

A further composition analysis as a percentage based on the initial sample component are presented in Table 2. As being widely reported [8,37], CCL in this work extracted most of the lignin $(86.02 \%)$, while $95.29 \%$ of the initial glucose, $25.80 \%$ of the initial xylose and only $9.73 \%$ of the initial lignin were remained in the CCL solid residue. In comparison, the CLL treatment further improved the fractionation efficiency, as $98.40 \%$ of the initial glucose, $24.10 \%$ of the initial xylose and $67.01 \%$ of the initial mannose were remained in the CLL solid residue, while only $7.03 \%$ of initial lignin was 
preserved. A promising $90.13 \%$ of the native lignin was extracted into CLL lignin phase, and the extracted DES lignin could be easily collected by precipitation with water. It was clearly shown that the replacement of chloride anion with lactate anion in DES promoted the separation selectivity during the DES treatment process of lignocellulosic biomass, which elevated the lignin extraction rate from $86.02 \%$ (CCL lignin) to $90.13 \%$ (CLL lignin). More important, only trace amount of glucose $(0.10 \%)$ was detected in the final CLL lignin.

Table 2. Chemical compositions of DES solid residues and DES lignin (as a percentage based on the initial sample component).

\begin{tabular}{ccccccc}
\hline & Glucose & Xylose & Mannose & Galactose & Arabinose & Lignin \\
\hline CCL solid residue & $95.29 \pm 3.42$ & $25.80 \pm 1.11$ & - & - & - & $9.73 \pm 0.78$ \\
CCL lignin & $0.17 \pm 0.1$ & - & - & - & - & $86.02 \pm 1.58$ \\
CLL solid residue & $98.40 \pm 0.42$ & $24.10 \pm 1.15$ & $67.01 \pm 2.21$ & - & - & $7.03 \pm 0.58$ \\
CLL lignin & $0.10 \pm 0.1$ & - & - & - & - & $90.13 \pm 2.63$ \\
\hline
\end{tabular}

All the results above clearly indicated that both CCL and CLL treatment could efficiently fractionate lignocellulosic biomass, as the glucose remained in the DES solid residue and most of lignin were extracted out as DES lignin. Between these DES treatment methods, CLL presented higher efficiency and selectivity, as better preservation of cellulose in the initial sample and higher lignin recovery rate were viewed in the CLL-treated sample. Therefore, it seems that the replacement of chloride anion in CCL with lactate anion (CLL) facilitated the fractionation of biomass, by extracting more lignin into DES lignin phase and leaving more carbohydrates in solid residue.

Indeed, even for the CLL treatment, there were still around $3 \%$ of lignin, $1 \%$ of glucose, $75 \%$ of xylose, $33 \%$ of mannose and almost all of the galactose and arabinose remained in the aqueous solutions, which may due to the thermal degradation and acid hydrolysis of hemicellulose under acid conditions $[8,38,39]$. However, these degraded carbohydrates presented limited impact on the DES recovery. They could be easily recovered by collecting the effluent after solvent (water and ethanol) washing, where up to $98 \%$ of the DES could be recovered.

\subsection{Characterization of Lignin}

\subsubsection{Lignin Purity}

For the composition analysis of lignin extracted by the DES treatment, the acid insoluble lignin (AIL), acid soluble lignin (ASL) and sugar content were measured. The results are presented as a percentage based on the testing sample and shown in Table 3. Lignin extracted by both CCL and CLL treatments were of high purity $(>85 \%)$, with only trace of glucose and no detectable hemicellulose residues. Lignin extracted by CCL (CCL lignin) was of $88.82 \%$ purity, with only $0.35 \%$ of glucose, while lignin extracted by CLL (CLL lignin) presented a higher purity $(91.17 \%)$ with only $0.21 \%$ of glucose detected. The lignin purity analysis results further supported our hypothesis that the removal of halogen anion could improve the lignin extraction efficiency, as the choline lactate-lactic acid DES not only extracted more lignin from the biomass, but also provided CLL lignin with higher purity.

Table 3. Purity of the DES lignin extracted from poplar as a percentage based on the testing sample.

\begin{tabular}{|c|c|c|c|c|c|c|c|c|}
\hline & Glucose & Xylose & Mannose & Galactose & Arabinose & ASL & AIL & Lignin \\
\hline Poplar & $45.46 \pm 1.21$ & $14.08 \pm 0.37$ & $1.71 \pm 0.19$ & $0.41 \pm 0.10$ & $0.21 \pm 0.10$ & 5.05 & 20.57 & $25.62 \pm 0.32$ \\
\hline CCL lignin & $0.35 \pm 0.10$ & - & - & - & - & 2.30 & 86.52 & $88.82 \pm 1.25$ \\
\hline CLL lignin & $0.21 \pm 0.10$ & - & - & - & - & 1.92 & 89.25 & $91.17 \pm 2.15$ \\
\hline
\end{tabular}

ASL—acid soluble lignin; AIL—acid insoluble lignin. 


\subsubsection{Molecular Weight Distribution}

The variation of weight average molecular weight $(\mathrm{Mw})$, number average molecular weight $(\mathrm{Mn})$ and the polydispersity index (PDI) during DES treatment are shown in Table 4. In comparison with the MWL lignin, DES extraction significantly reduced the Mw and Mn value of lignin. Mw and Mn of CCL lignin decreased from 10,000 and 4166 to 4416 and $2349 \mathrm{~g} / \mathrm{mol}$, respectively. At the same time, Mw and Mn of the CLL lignin could further reduce to 1805 and $971 \mathrm{~g} / \mathrm{mol}$, respectively. This indicated that both DES treatment could facile the cleavage of the lignin linkage. It should be noticed that the molecular weights of DES lignin in this work were also lower than those reported in literature extracted through organic solvents [40]. Thereby, in this work the DES lignin extracted from poplar presented unique low molecular weight, which could upgrade the value of lignin stream and facilitate further valorization. The CLL treatment provided lignin stream of a much lower molecular weight, which could attribute to a stronger or more interactions formed between the DES and lignin molecules.

In addition, it was shown in Table 4 that a more concentrated distribution of polydispersity of lignin was found with DES treatment. Both DES treatment resulted in a narrow molecular weight distribution (PDI $<2.0$ ), while the PDI of MWL is 2.40. CLL lignin was capable to provide lignin stream with better uniformity (PDI $=1.86$ ), which may benefit the downstream process. Thus, it verified that CLL treatments was a promising method for lignin extraction.

Table 4. Average molecular weights and polydispersity indices of MWL and DES lignin.

\begin{tabular}{cccc}
\hline & Mw (g/mol) & Mn $(\mathrm{g} / \mathbf{m o l})$ & PDI \\
\hline Lit. MWL * & 10,000 & 4166 & 2.40 \\
MWL & 6374 & 5542 & 1.15 \\
CCL lignin & 4416 & 2349 & 1.88 \\
CLL lignin & 1805 & 971 & 1.86 \\
\hline \multicolumn{3}{c}{ * Literature MWL data taken from Li, N. et al. 2018 [41]. }
\end{tabular}

The results in Table 4 illustrate that the choline-based DES treatment could selectively extract lignin from poplar not only with high purity, but also with low molecular weight and good uniformity. CLL, which was obtained by replacing chloride anion in CCL with lactate anion, facilitated the cleavage of intermolecular linkage. The lignin may form a stronger interaction with CLL and degraded into smaller molecules during the DES treating process. As a result, the selectivity of lignin extraction improved and CLL lignin with higher purity, lower molecular weight and more concentrated distribution obtained. These lignins extracted with appropriate molecular weight were ideal for the downstream productions [6].

\subsection{Structure Characterization of Lignin}

\subsubsection{FT-IR Analysis}

The FT-IR analysis of the initial sample, MWL and DES lignin are shown in Figure 3. Characteristic peaks of different bonds [8,42] and detailed signal assignments are listed in Table 5. The FT-IR analysis confirmed a high purity lignin was extracted by DES treatment, as both CLL and CCL lignin presented similar absorbance bands with those of MWL. Typical lignin benzene ring skeleton bands were found at 1604 and $1510 \mathrm{~cm}^{-1}$ in all lignin samples, illustrating a well-preserved lignin framework after DES treatment. Absorption bands at 1328 and $1220 \mathrm{~cm}^{-1}$ were corresponded to $\mathrm{C}-\mathrm{O}$ stretching vibration in syringyl units, while the absorbance at $1116 \mathrm{~cm}^{-1}$ was related to the $\mathrm{C}-\mathrm{H}$ stretching vibration. All these characteristic peaks were found in the MWL, CLL lignin and CCL lignin samples, indicating the syringyl unit was left intact after both DES extraction. The absorption at 1270 and $1032 \mathrm{~cm}^{-1}$ were associated with the $\mathrm{C}-\mathrm{O}$ stretch and $\mathrm{C}-\mathrm{H}$ bending vibration of guaiacyl unit, respectively. The feature of GS-typed lignin was demonstrated in the FT-IR spectra. It was noticed that the guaiacyl unit-related peaks presented lower intensities after both DES treatments, illustrating the cleavage of guaiacyl units 
occurred during the DES extraction. Therefore, the lignin depolymerization reported in GPC results may possibly attribute to the degradation of guaiacyl units, while the syringyl units and the aromatic ring remain unchanged.

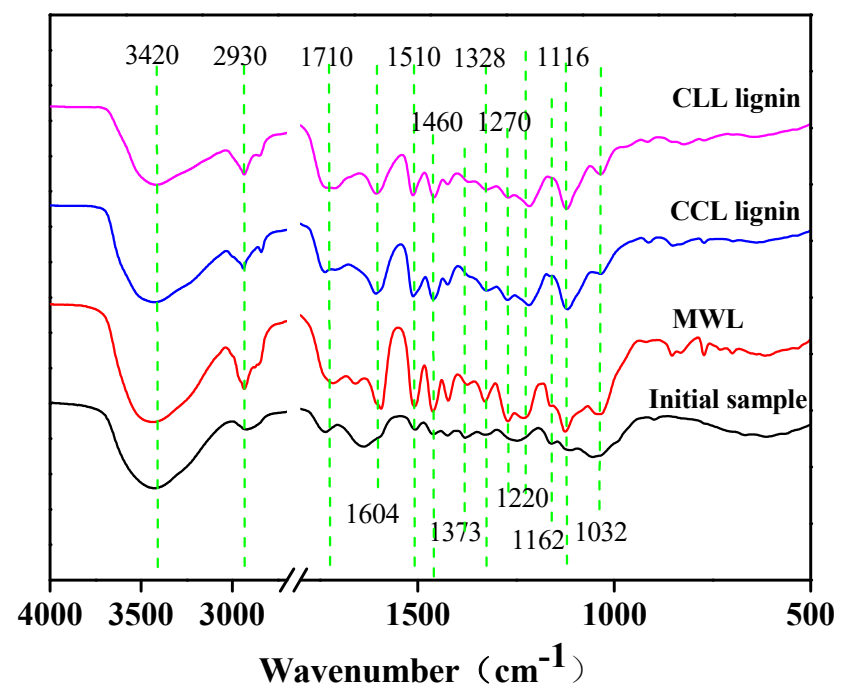

Figure 3. FT-IR analysis of the initial sample milled wood lignin (MWL), CLL lignin and CCL lignin.

Table 5. Assignment of FT-IR spectra of the initial sample, MWL, CLL lignin and CCL lignin.

\begin{tabular}{cccccc}
\hline $\begin{array}{c}\text { Wavenumbers } \\
\left(\mathbf{c m}^{-1} \mathbf{)}\right.\end{array}$ & Assignment (Bond) & $\begin{array}{c}\text { Initial } \\
\text { Sample }\end{array}$ & $\begin{array}{c}\text { CLL } \\
\text { Lignin }\end{array}$ & $\begin{array}{c}\text { CCL } \\
\text { Lignin }\end{array}$ & MWL \\
\hline 3420 & O-H stretching vibration & $\sqrt{ }$ & $\sqrt{ }$ & $\sqrt{ }$ & $\sqrt{ }$ \\
2930 & C-H stretching vibration in methyl & $\sqrt{ }$ & $\sqrt{ }$ & $\sqrt{ }$ & $\sqrt{ }$ \\
1710 & C=O stretching vibration & $\sqrt{ }$ & $\sqrt{ }$ & $\sqrt{ }$ & $\sqrt{ }$ \\
1604,1510 & Aromatic ring skeleton vibration & $\sqrt{ }$ & $\sqrt{ }$ & $\sqrt{ }$ & $\sqrt{ }$ \\
1460 & C-H deformation vibration in -CH $2^{-}$ & $\sqrt{ }$ & $\sqrt{ }$ & $\sqrt{ }$ & $\sqrt{ }$ \\
1373 & C-H bending vibration of aliphatic compounds in carbohydrate & $\sqrt{ }$ & $\times$ & $\times$ & $\times$ \\
1328,1220 & C-O stretching vibration of syringyl units & $\sqrt{ }$ & $\sqrt{ }$ & $\sqrt{ }$ & $\sqrt{ }$ \\
1270 & C-O stretching vibration of guaiacyl units & $\sqrt{ }$ & $\sqrt{ }$ & $\sqrt{ }$ & $\sqrt{ }$ \\
1162 & C-O-C symmetrical stretching vibration in carbohydrate & $\sqrt{ }$ & $\times$ & $\times$ & $\times$ \\
1116 & C-H stretching vibration of syringyl units & $\sqrt{ }$ & $\sqrt{ }$ & $\sqrt{ }$ & $\sqrt{ }$ \\
1032 & C-H bending vibration of guaiacyl units & $\sqrt{ }$ & $\sqrt{ }$ & $\sqrt{ }$ & $\sqrt{ }$ \\
\hline
\end{tabular}

In addition, the signals of carbohydrate bands at 1373 and $1162 \mathrm{~cm}^{-1}$ did not appear in both CCL and CLL lignin samples, while these carbohydrate characteristic peaks appeared clearly in initial sample. This confirmed a successfully selective lignin extraction by the DES treatment, which could provide valuable lignin stream with only limited hydrocarbons contaminations.

\subsubsection{HSQC NMR Analysis}

The structural analysis and comparation of DES lignin and MWL were investigated by HSQC NMR; the results are shown in Figure 4. Assignment of the peaks in the spectra were based on previous report [37]. The peaks corresponding to $\beta$-aryl ether connections are shown for $A_{\alpha}$ $\left(\delta_{\mathrm{C}} / \delta_{\mathrm{H}} 4.95 / 69.92 \mathrm{ppm}\right), \mathrm{A}_{\gamma}\left(\delta_{\mathrm{C}} / \delta_{\mathrm{H}} 3.81 / 61.69 \mathrm{ppm}\right), \mathrm{A}_{\gamma}^{\prime}\left(\delta_{\mathrm{C}} / \delta_{\mathrm{H}} 4.67 / 64.85 \mathrm{ppm}\right), \mathrm{A}(\mathrm{H} / \mathrm{G})_{\beta}\left(\delta_{\mathrm{C}} / \delta_{\mathrm{H}}\right.$ $4.63 / 80.48 \mathrm{ppm})$ and $\mathrm{A}(\mathrm{S})_{\beta}\left(\delta_{\mathrm{C}} / \delta_{\mathrm{H}} 4.88 / 78.74 \mathrm{ppm}\right) . \beta-\beta$ connections are shown for $\mathrm{B}_{\alpha}\left(\delta_{\mathrm{C}} / \delta_{\mathrm{H}}\right.$ 4.70/85.49 ppm), $\mathrm{B}_{\beta}\left(\delta_{\mathrm{C}} / \delta_{\mathrm{H}} 3.06 / 53.71 \mathrm{ppm}\right)$ and $\mathrm{B}_{\gamma}\left(\delta_{\mathrm{C}} / \delta_{\mathrm{H}} 3.86 / 71.80 \mathrm{ppm}\right) . \beta-5$ connections are shown for $C_{\alpha}\left(\delta_{C} / \delta_{H} 5.59 / 87.55 \mathrm{ppm}\right), C_{\beta}\left(\delta_{C} / \delta_{H} 3.76 / 59.36\right)$ and $C_{\gamma}\left(\delta_{C} / \delta_{H} 4.36 / 65.09 \mathrm{ppm}\right)$. Moreover, methoxyl connections was shown at $\left(\delta_{C} / \delta_{\mathrm{H}} 3.76 / 56.33 \mathrm{ppm}\right)$. As shown in Figure 4 , the MWL had abundant $\beta$-ether bond units (Figure $4 \mathrm{~d}-\mathrm{f}$ ) as well as a small amount of resinol (Figure $4 \mathrm{~h}$ ) and phenylcoumarin (Figure 4i), which was in agreement with our previous FT-IR results, where the poplar lignin demonstrated typical GS-typed lignin. 
(a)

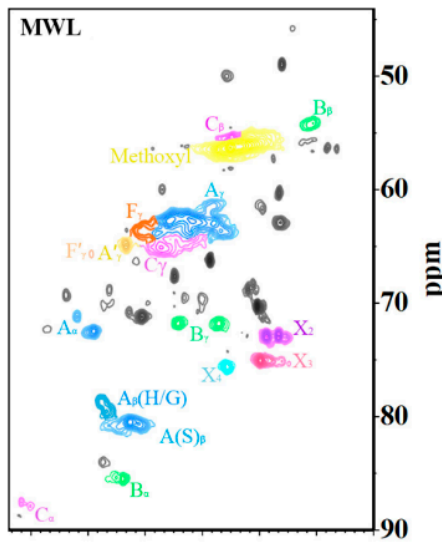

$\begin{array}{lllllll}5.5 & 5.0 & 4.5 & 4.0 & 3.5 & 3.0 & 2.5\end{array}$ ppm (b)

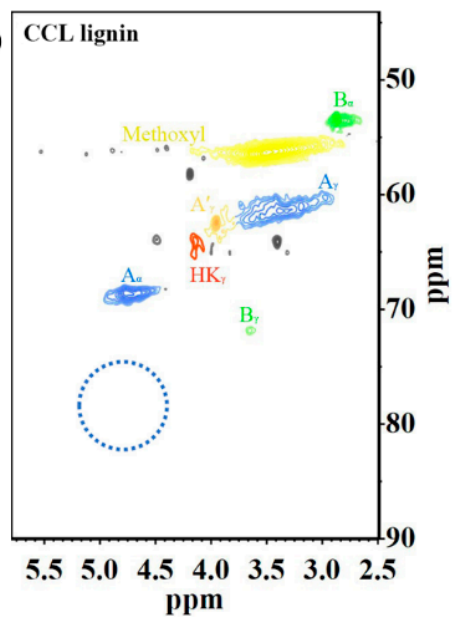

(c)

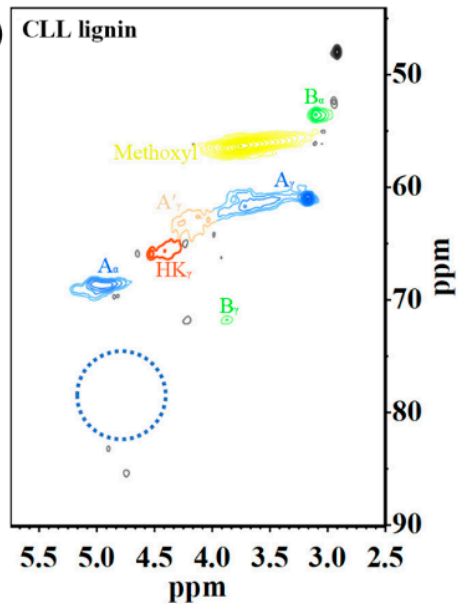

(d)

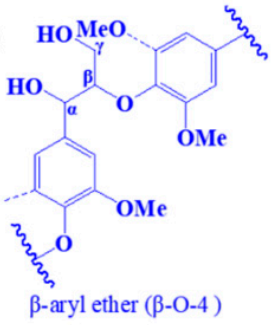

A

(h)

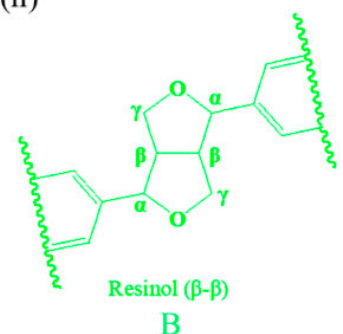

(e)

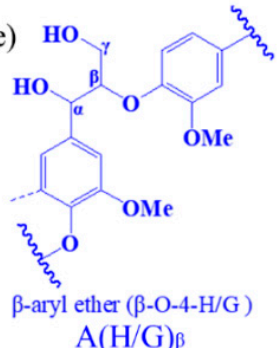

(i)

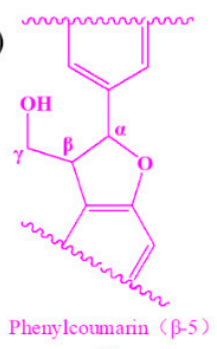

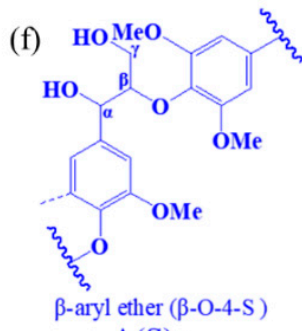
$\mathrm{A}(\mathrm{S}) \mathrm{B}$

(k)

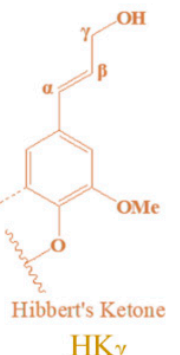

(g)

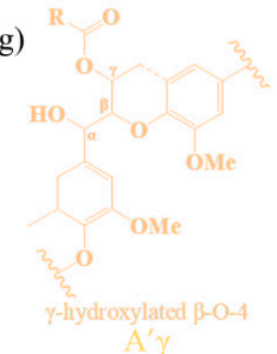

(1)

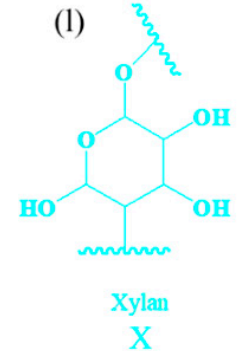

Figure 4. HSQC NMR analysis of MWL and DES lignin. (a) milled wood lignin; (b) lignin extracted by choline chloride-lactic acid; (c) lignin extracted by choline chloride-lactic acid; (d) $\beta$-O-4 aryl ether bond; (e) $\beta-\mathrm{O}-4-\mathrm{H} / \mathrm{G}$ aryl ether bond; (f) $\beta-\mathrm{O}-4-\mathrm{S}$ aryl ether bond; (g) hydroxylated $\beta-\mathrm{O}-4$; (h) resibol; (i) Phenylcomarin; (k) Hibbert's ketone; (1) xylan. The abbreviation of A, A(H/G) $\beta, A(S) \beta, A^{\prime} \gamma, B, C$, $\mathrm{HK} \gamma$ and $\mathrm{X}$ listed in Figure $4 \mathrm{~d}-\mathrm{l}$ are used to indicate the different types of lignin structure shown in Figure $4 \mathrm{a}-\mathrm{c}$.

It can be seen from Figure $4 \mathrm{~b}, \mathrm{c}$ that the absence of major contours corresponding to the non-anomeric carbohydrates $\left(\delta_{\mathrm{C}} / \delta_{\mathrm{H}} 65-85 / 2.5-4.5 \mathrm{ppm}\right)$ in both CCL and CLL were noticed, which verified the excellent lignin extraction selectivity. What is more, after both DES treatment $\beta$-ether bond $\left(\mathrm{A}_{\alpha}\right.$ and $\left.\mathrm{A}_{\gamma}\right)$ was kept, however $\beta-\mathrm{O}-4$ ether linkages connected to guaiacyl (Figure 4e) and syringyl (Figure $4 \mathrm{f}$ ) could not be detected in the HSQC spectrum (blue cycle), which indicated that a cleavage of $\beta-\mathrm{O}-4$ ether bond between the guaiacyl and syringyl structural units occurred during the DES treatment. It was reported that lignin degradation in DES was similar to the typical lignin acidolysis, during which Hibbert's ketone was generated as intermediate $[41,43,44]$. As shown in Figure 4, the presence of Hibbert's ketone (Figure 4k), 4.21/67.5 ppm) was detected in both CCL and CLL lignin samples, which verified the cleavage of $\beta-\mathrm{O}-4$ bonds. Therefore, the HSQC analysis confirmed that DES treatment in this work facilitated the cleavage of $\beta-\mathrm{O}-4$ bonds in lignin macromolecules and promoted the lignin extraction. 
It could also be seen that after both DES pretreatment, most of the $\mathrm{C}-\mathrm{C}$ bonds were retained and remained as the main interunit bonds in lignin samples, while the presence of ether bonds significantly reduced. Hence, ether bonds are more vulnerable to damage than carbon-carbon bonds [7], the decrease of ether bonds in DES lignin could provide lignin stream with an improved polymer stability and utility value.

The characterizations of CLL lignin and CCL lignin interestingly presented similar composition and structure, which indicated both DES interacted with lignin macromolecule in the same mechanism of action. It was proposed in this work that DES treatment induced the cleavage of the $\beta-\mathrm{O}-4$ bonds in lignin, thereby degrading the lignin macromolecule into smaller molecules, facilitating the fractionation of lignocellulosic biomass. A possible mechanism was that the replacement of chloride anion with lactate anion in DES system allowing CLL to present stronger or more interactions with lignin during DES treating process. A typical reaction for the cleavage of lignin $\beta-\mathrm{O}-4$ bonds is shown in Figure 5, in which the formation of protonated intermediates was reported [45]. Different nucleophilicities of the anion in DES altered the cation-anion interaction, consequently influenced the solvation of the protonated intermediates [46]. In this case, chloride anion was of strong electronegativity, thereby presenting strong cation-anion interaction and a weak interaction. In contrast, lactate anion with less electronegativity presented weak cation-anion association, but with strong interaction. This allowed CLL in the mixture to help with the stabilization of the protonated intermediates by forming hydrogen bonds. Therefore, the CLL could serve as a better catalyst during the cleavage of $\beta-\mathrm{O}-4$ bonds in lignin degradation, promoting the selective extraction of lignin compounds from lignocellulosic biomass. This could contribute to the production of lignin streams with high yield, high purity, low molecular weight and good uniformity after CLL treatment, which were also more suitable for the downstream production of biofuels and functional materials.

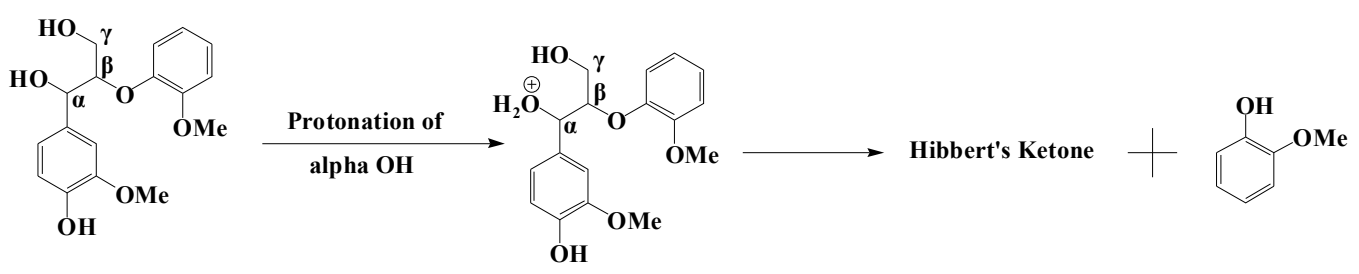

Figure 5. Mechanism of $\beta-\mathrm{O}-4$ ether link cleavage of lignin compounds [45].

\section{Conclusions}

CLL as a deep eutectic solvent (DES) with low halogen content was applied to enhance the lignin extraction of poplar. The replacement of chloride anion with lactate anion from CCL can reduce the potential corrosion to the equipment and also allowed CLL to present a significantly high efficiency in fractionating poplar biomass. A better preservation of glucan in the DES solid residue and a higher extraction yield of lignin in the DES lignin were obtained in the CLL treatment process. In addition, DES lignin with higher purity, lower molecular weight and more concentrated distribution were also achieved in the CLL treatment. The choline-based DES in this work could cleavage the $\beta-\mathrm{O}-4$ bonds of guaiacyl units in lignin macromolecule, thus, breaking them into smaller molecules, as a result facilitating the lignin extraction process. The removal of chloride anion allowed the CLL acted as a more efficient DES interacting with lignin macromolecules, hence providing CLL lignin with higher uniformity and suitable molecular weight. The low halogen content DES system proposed in present work could improve the fractionation of lignocellulosic biomass, providing lignin stream as promising feedstock for downstream process, thereby benefiting the future large-scale industrial process.

Author Contributions: J.L. and L.Q. have contributed equally to this work. L.Q., G.Y. and J.C. conceived and designed the experiments; J.L., L.Q. and Y.X. performed the experiments; J.L., L.Q., M.H. and Y.X. analyzed the data; G.Y. and Y.X. contributed reagents/materials/analysis tools; L.Q. and J.L. wrote the study; G.Y., L.A.L. and J.C. reviewed the manuscript and made comments. All authors have read and agreed to the published version of the manuscript. 
Funding: The authors are grateful for the financial support received from the National Natural Science Foundation of China (Grant No. 31770628, 31901267), the Provincial Key Research and Development Program of Shandong (Grant No. 2019JZZY010326, 2019JZZY010328), Foundation of State Key Laboratory of Biobased Material and Green Papermaking (No. ZZ20190214) and the Taishan Scholars Program.

Acknowledgments: The authors are grateful for the financial support received from the National Natural Science Foundation of China (Grant No. 31770628, 31901267), the Provincial Key Research and Development Program of Shandong (Grant No. 2019JZZY010326, 2019JZZY010328), Foundation of State Key Laboratory of Biobased Material and Green Papermaking (No. ZZ20190214) and the Taishan Scholars Program.

Conflicts of Interest: The authors declare no conflicts of interest.

\section{References}

1. Elgharbawy, A.A.; Hayyan, M.; Hayyan, A.; Basirun, W.J.; Salleh, H.M.; Mirghani, M.E. A grand avenue to integrate deep eutectic solvents into biomass processing. Biomass Bioenergy 2020, 137, 105550. [CrossRef]

2. Stafford, W.; De Lange, W.; Nahman, A.; Chunilall, V.; Lekha, P.; Andrew, J.; Johakimu, J.; Sithole, B.; Trotter, D. Forestry biorefineries. Renew. Energy 2020, 154, 461-475. [CrossRef]

3. Stewart, D. Lignin as a base material for materials applications: Chemistry, application and economics. Ind. Crop. Prod. 2008, 27, 202-207. [CrossRef]

4. Alvira, P.; Tomás-Pejó, E.; Ballesteros, M.; Negro, M.J. Pretreatment technologies for an efficient bioethanol production process based on enzymatic hydrolysis: A review. Bioresour. Technol. 2010, 101, 4851-4861. [CrossRef] [PubMed]

5. Satari, B.; Karimi, K.; Kumar, R. Cellulose solvent-based pretreatment for enhanced second-generation biofuel production: A review. Sustain. Energy Fuels 2019, 3, 11-62. [CrossRef]

6. Guo, Z.; Zhang, Q.; You, T.; Zhang, X.; Xu, F.; Wu, Y.-Y. Short-time deep eutectic solvent pretreatment for enhanced enzymatic saccharification and lignin valorization. Green Chem. 2019, 21, 3099-3108. [CrossRef]

7. Alvarez-Vasco, C.; Ma, R.; Quintero, M.; Guo, M.; Geleynse, S.; Ramasamy, K.K.; Wolcott, M.P.; Zhang, X. Unique low-molecular-weight lignin with high purity extracted from wood by deep eutectic solvents (DES): A source of lignin for valorization. Green Chem. 2016, 18, 5133-5141. [CrossRef]

8. Li, T.; Lyu, G.; Liu, Y.; Lou, R.; Lucia, L.A.; Yang, G.; Chen, J.; Saeed, H.A. Deep Eutectic Solvents (DESs) for the Isolation of Willow Lignin (Salix matsudana cv. Zhuliu). Int. J. Mol. Sci. 2017, 18, 2266. [CrossRef]

9. An, Y.-X.; Zong, M.-H.; Wu, H.; Li, N. Pretreatment of lignocellulosic biomass with renewable cholinium ionic liquids: Biomass fractionation, enzymatic digestion and ionic liquid reuse. Bioresour. Technol. 2015, 192, 165-171. [CrossRef] [PubMed]

10. Himmel, M.E.; Ding, S.-Y.; Johnson, D.K.; Adney, W.S.; Nimlos, M.R.; Brady, J.W.; Foust, T.D. Biomass Recalcitrance: Engineering Plants and Enzymes for Biofuels Production. Science 2007, 315, 804-807. [CrossRef] [PubMed]

11. Jiang, Z.; Hu, C. Selective extraction and conversion of lignin in actual biomass to monophenols: A review. J. Energy Chem. 2016, 25, 947-956. [CrossRef]

12. Kumar, A.K.; Sharma, S. Recent updates on different methods of pretreatment of lignocellulosic feedstocks: A review. Bioresour. Bioprocess. 2017, 4, 278. [CrossRef]

13. Al Arni, S. Extraction and isolation methods for lignin separation from sugarcane bagasse: A review. Ind. Crop. Prod. 2018, 115, 330-339. [CrossRef]

14. Xu, H.; Peng, J.; Kong, Y.; Liu, Y.; Su, Z.; Li, B.; Tian, W. Key process parameters for deep eutectic solvents pretreatment of lignocellulosic biomass materials: A review. Bioresour. Technol. 2020, 123416. [CrossRef] [PubMed]

15. Abbott, A.P.; Capper, G.; Davies, D.L.; Rasheed, R.K.; Tambyrajah, V. Novel solvent properties of choline chloride/urea mixturesElectronic supplementary information (ESI) available: Spectroscopic data. Chem. Commun. 2003, 9, 70-71. Available online: http://www.rsc.org/suppdata/cc/b2/b210714g/ (accessed on 10 July 2020). [CrossRef] [PubMed]

16. Smith, E.L.; Abbott, A.P.; Ryder, K.S. Deep Eutectic Solvents (DESs) and Their Applications. Chem. Rev. 2014, 114, 11060-11082. [CrossRef]

17. Zhang, Q.; Vigier, K.D.O.; Royer, S.; Jérôme, F. Deep eutectic solvents: Syntheses, properties and applications. Chem. Soc. Rev. 2012, 41, 7108-7146. [CrossRef] 
18. Dai, Y.; Van Spronsen, J.; Witkamp, G.-J.; Verpoorte, R.; Choi, Y.H. Natural deep eutectic solvents as new potential media for green technology. Anal. Chim. Acta 2013, 766, 61-68. [CrossRef]

19. Francisco, M.; Bruinhorst, A.V.D.; Kroon, M.C. New natural and renewable low transition temperature mixtures (LTTMs): Screening as solvents for lignocellulosic biomass processing. Green Chem. 2012, 14, 2153-2157. [CrossRef]

20. Procentese, A.; Johnson, E.; Orr, V.; Campanile, A.G.; Wood, J.A.; Marzocchella, A.; Rehmann, L. Deep eutectic solvent pretreatment and subsequent saccharification of corncob. Bioresour. Technol. 2015, 192, 31-36. [CrossRef] [PubMed]

21. Zhang, C.-W.; Xia, S.; Ma, P.-S. Facile pretreatment of lignocellulosic biomass using deep eutectic solvents. Bioresour. Technol. 2016, 219, 1-5. [CrossRef] [PubMed]

22. Kumar, A.K.; Parikh, B.S.; Pravakar, M. Natural deep eutectic solvent mediated pretreatment of rice straw: Bioanalytical characterization of lignin extract and enzymatic hydrolysis of pretreated biomass residue. Environ. Sci. Pollut. Res. 2015, 23, 9265-9275. [CrossRef] [PubMed]

23. Brandt-Talbot, A.; Gräsvik, J.; Hallett, J.P.; Welton, T. Deconstruction of lignocellulosic biomass with ionic liquids. Green Chem. 2013, 15, 550-583. [CrossRef]

24. Ninomiya, K.; Yamauchi, T.; Kobayashi, M.; Ogino, C.; Shimizu, N.; Takahashi, K. Cholinium carboxylate ionic liquids for pretreatment of lignocellulosic materials to enhance subsequent enzymatic saccharification. Biochem. Eng. J. 2013, 71, 25-29. [CrossRef]

25. Ninomiya, K.; Inoue, K.; Aomori, Y.; Ohnishi, A.; Ogino, C.; Shimizu, N.; Takahashi, K. Characterization of fractionated biomass component and recovered ionic liquid during repeated process of cholinium ionic liquid-assisted pretreatment and fractionation. Chem. Eng. J. 2015, 259, 323-329. [CrossRef]

26. Liu, Q.-P.; Hou, X.-D.; Li, N.; Zong, M.-H. Ionic liquids from renewable biomaterials: Synthesis, characterization and application in the pretreatment of biomass. Green Chem. 2012, 14, 304-307. [CrossRef]

27. Hou, X.-D.; Smith, T.J.; Li, N.; Zong, M.-H. Novel renewable ionic liquids as highly effective solvents for pretreatment of rice straw biomass by selective removal of lignin. Biotechnol. Bioeng. 2012, 109, $2484-2493$. [CrossRef]

28. Hou, X.-D.; Li, N.; Zong, M.-H. Facile and Simple Pretreatment of Sugar Cane Bagasse without Size Reduction Using Renewable Ionic Liquids-Water Mixtures. ACS Sustain. Chem. Eng. 2013, 1, 519-526. [CrossRef]

29. Hou, X.-D.; Li, N.; Zong, M.-H. Significantly enhancing enzymatic hydrolysis of rice straw after pretreatment using renewable ionic liquid-water mixtures. Bioresour. Technol. 2013, 136, 469-474. [CrossRef]

30. Hou, X.-D.; Xu, J.; Li, N.; Zong, M.-H. Effect of anion structures on cholinium ionic liquids pretreatment of rice straw and the subsequent enzymatic hydrolysis. Biotechnol. Bioeng. 2014, 112, 65-73. [CrossRef]

31. Hou, X.-D.; Liu, Q.-P.; Smith, T.J.; Li, N.; Zong, M.-H. Evaluation of Toxicity and Biodegradability of Cholinium Amino Acids Ionic Liquids. PLoS ONE. 2013, 8, e59145. [CrossRef] [PubMed]

32. Ferguson, J.L.; Holbrey, J.D.; Ng, S.; Plechkova, N.V.; Seddon, K.R.; Tomaszowska, A.A.; Wassell, D.F. A greener, halide-free approach to ionic liquid synthesis. Pure Appl. Chem. 2011, 84, 723-744. [CrossRef]

33. Muhammad, N.; Hossain, M.I.; Man, Z.; El-Harbawi, M.; Bustam, M.A.; Noaman, Y.A.; Alitheen, N.B.M.; Ng, M.K.; Hefter, G.; Yin, C.-Y. Synthesis and Physical Properties of Choline Carboxylate Ionic Liquids. J. Chem. Eng. Data. 2012, 57, 2191-2196. [CrossRef]

34. Sluiter, A.; Hames, B.; Ruiz, R.; Scarlata, C.; Sluiter, J.; Templeton, D.; Crocker, D. Determination of Structural Carbohydrates and Lignin in Biomass. Laboration Anal. Proced. 2008, 1617, 1-16.

35. Peng, J.; Yang, G.; Qi, L.; Liu, J.; Li, F.; Chen, J.; Lucia, L.A. Enhancement of Delignification by Ionic Liquids Pretreatment and Modification of Hardwood Kraft Pulp in Preparation for Bleaching. BioRes. 2020, 15, 6299-6308. [CrossRef]

36. Björkman, A. Studies on finely divided wood. Part 1. Extraction of lignin with neutral solvents. Sven. Papperst 1956, 59, 477-485.

37. Lyu, G.; Li, T.; Ji, X.; Yang, G.; Liu, Y.; Lucia, L.; Chen, J. Characterization of Lignin Extracted from Willow by Deep Eutectic Solvent Treatments. Polymers 2018, 10, 869. [CrossRef]

38. Li, L.F.; Hu, Y.C. Application of ionic liquids in lignin processing. Chem. Ind. For. Prod. 2015, 35, $163-170$. [CrossRef]

39. Sun, Y.-C.; Xu, J.-K.; Xu, F.; Sun, S. Efficient separation and physico-chemical characterization of lignin from eucalyptus using ionic liquid-organic solvent and alkaline ethanol solvent. Ind. Crop. Prod. 2013, 47, 277-285. [CrossRef] 
40. Jääskeläinen, A.-S.; Liitiä, T.; Mikkelson, A.; Tamminen, T. Aqueous organic solvent fractionation as means to improve lignin homogeneity and purity. Ind. Crop. Prod. 2017, 103, 51-58. [CrossRef]

41. Li, N.; Li, Y.; Yoo, C.G.; Yang, X.; Lin, X.; Ralph, J.; Pan, X. An uncondensed lignin depolymerized in the solid state and isolated from lignocellulosic biomass: A mechanistic study. Green Chem. 2018, 20, 4224-4235. [CrossRef]

42. Zhou, Q.; Chen, J.; Wang, C.; Yang, G.; Ji, X.; Peng, J.; Xu, F. Quantitative structures and thermal properties of Miscanthus $\times$ giganteus lignin after alcoholamine-based ionic liquid pretreatment. Ind. Crop. Prod. 2020, 147, 112232. [CrossRef]

43. Brandt, A.; Welton, T.; Chen, L.; Van Dongen, B.E.; Hallett, J.P. Structural changes in lignins isolated using an acidic ionic liquid water mixture. Green Chem. 2015, 17, 5019-5034. [CrossRef]

44. Miles-Barrett, D.M.; Neal, A.R.; Hand, C.; Montgomery, J.R.D.; Panovic, I.; Ojo, O.S.; Lancefield, C.S.; Cordes, D.B.; Slawin, A.M.Z.; Lebl, T.; et al. The synthesis and analysis of lignin-bound Hibbert ketone structures in technical lignins. Org. Biomol. Chem. 2016, 14, 10023-10030. [CrossRef]

45. De Gregorio, G.F.; Weber, C.C.; Gräsvik, J.; Welton, T.; Brandt, A.; Hallett, J.P. Mechanistic insights into lignin depolymerisation in acidic ionic liquids. Green Chem. 2016, 18, 5456-5465. [CrossRef]

46. Jia, S.; Cox, B.J.; Guo, X.; Zhang, Z.C.; Ekerdt, J.G. Cleaving the $\beta-\mathrm{O}-4$ Bonds of Lignin Model Compounds in an Acidic Ionic Liquid, 1-H-3-Methylimidazolium Chloride: An Optional Strategy for the Degradation of Lignin. ChemSusChem 2010, 3, 1078-1084. [CrossRef]

(C) 2020 by the authors. Licensee MDPI, Basel, Switzerland. This article is an open access article distributed under the terms and conditions of the Creative Commons Attribution (CC BY) license (http://creativecommons.org/licenses/by/4.0/). 